\title{
Katastrophenschutzrecht - Besichtigung eines verdrängten Rechtsgebiets
}

\section{Katastrophen als Diskontinuitätsereignisse}

Der Begriff der Katastrophe markiert eine soziale Grenzsituation, zumindest für die Betroffenen. Eine Katastrophe ist das Diskontinuitätsereignis schlechthin, in dem basale Erwartungen enttäuscht und Routinen entwertet werden: Man wird von der geliebten Person verlassen, der Arbeitsplatz wird verloren, das Haus von Flut fortgespült, ein großtechnisches System versagt, ein terroristischer Anschlag entwertet basale Sicherheitserwartungen. Man kann nicht vorhersehen, wann das passiert, welche Schäden auftreten, wer betroffen sein wird, obwohl man selbstverständlich weiß, dass alles dies passieren kann, aber das wird latent gehalten. Eine komplexe und für die Betroffenen meist nicht entschlüsselbare Hintergrunddynamik natürlicher Ereignisse und/oder gesellschaftlicher oder persönlicher Entscheidungen äußert sich in dem überraschenden Ereignis, das durch eine extreme Fallhöhe zu den persönlichen oder gesellschaftlichen Normalitätserwartungen gekennzeichnet ist. In diesem Sinne kann man davon sprechen, dass es dann, wenn eine für ein personales oder soziales System bestandswichtige Erwartung enttäuscht wird und diese Diskontinuität in wesentlichen Bezügen intransparent ist, zur Kommunikation über Katastrophen kommt, ${ }^{1}$ diese antizipierend oder reaktiv.

Dieser Bezug auf die Enttäuschung bestandswichtiger Erwartungen sagt über die Gründe nichts aus. Sie können in natürlichen Ereignissen oder Handlungen und Entscheidungen liegen, immer aber sind sie soziale Ereignisse, die als antizipierte oder eingetretene Katastrophe kommuniziert werden. Allerdings führt die Semantik der Katastrophe noch ältere Traditionen mit, die als Ursache von katastrophalen Entwicklungen Schicksal, Gott oder die Natur namhaft machten. ${ }^{2}$ Noch in der Redeweise von »Naturkatastrophen« schwingt diese Tradition mit, so als sei die Klimaveränderung und die mit ihr verbundenen Folgen nicht auch menschlichem Handeln und Entscheiden, wie vermittelt auch immer, zumindest teilweise zurechenbar. Die Rückführung auf Natur, wiewohl in Einzelfällen wohl begründet, ${ }^{3}$ suggeriert Unabänderbarkeit und Unbeeinflussbarkeit, dunkelt damit aber gesellschaftliche Entscheidungen, sozio-kulturelle und technische Faktoren ab. In diesem Sinne sind Katastrophen gesellschaftliche Konstruktionen. ${ }^{4}$ Nicht nur unterscheidet sich das, was als Katastrophe wahrge-

1 Japp, Zur Soziologie der Katastrophe, in: Clausen/Geenen/Macamo (Hrsg.), Entsetzliche soziale Prozesse, 2003, S. 77, 79.

2 Zur Entwicklung des Begriffs der Katastrophe Dombrowsky, Katastrophe und Katastrophenschutz, 1989; Kötter, Definitionen der Katastrophe in der Soziologie 1989-1998 in kritischer Zusammenfassung, 2001.

3 Ein Beispiel dafür wären Erdbeben, die freilich nur hinsichtlich der Ursachen, nicht aber der dadurch ausgelösten Schäden als gleichsam »echte « Naturkatastrophen anzusehen wären.

4 In der nüchternen Sprache der Versicherungsbranche: »Eine Woche vor dem Beben vor Sumatra gab es in der Antarktis ein Beben der Stärke 8,1. Da hat es vermutlich tausende Pinguine von den Füßen gerissen, aber für den Menschen war es keine Katastrophe.« Berz, Die Zeit - Wissen: Rechnen mit der Katastrophe. Allgemein zur sozialen Konstruktion von Risiken; vgl. Japp, Risiko, 2001 m. w. N. 
nommen wird, je nach sozialem und kulturellem Kontext: Auch innerhalb einer Gesellschaft ist das, was als Katastrophe wahrgenommen wird, unterschiedlich, etwa entlang von Linien der Stratifizierung der Gesellschaft: Was dem einen als Katastrophe gilt, weil es seine Existenzgrundlage vernichtet, ist dem anderen ein Versicherungsfall, der Raum für infrastrukturelle Modernisierungen schafft, also Opportunitäten hervorbringt. Mehr noch: Was als Katastrophe wahrgenommen wird, lässt sich ex ante nicht sicher vorhersehen und schafft Risiken (und Chancen) für das politische System. ${ }^{5}$ Oftmals kann eben nur in der nachträglichen Beobachtung im Spiegel der Massenmedien gesehen werden, dass andere einen Geschehensablauf als katastrophal wahrgenommen haben, das politische System aber von einem gleichsam normalen Schadensverlauf ausgegangen war. Insoweit unterscheidet sich die Wahrnehmung von Katastrophen nicht grundsätzlich von der Wahrnehmung und Einschätzung von Risiken. Auch für sie gilt, dass sie aufgrund der Pluralität möglicher Beobachtungsstandpunkte nicht in dem Sinne objektivierbar sind, dass an Zahl von Toten, Sachschaden etc. angeknüpft werden kann, um zu prognostizieren, was als Katastrophe ex ante wahrgenommen werden mag. ${ }^{6}$ Gleichwohl unterscheiden sich jedenfalls in der Antizipation Risiken und Katastrophen. Risiken sind dadurch gekennzeichnet, dass man Opportunitäten und Verluste - wie unsicher auch immer - balanciert, mögliche Katastrophen sind schlechthin zu vermeiden. ${ }^{7}$ Wenn das richtig ist, dann ist Katastrophenkommunikation gerade auf Vermeidung und - sofern nicht möglich - Begrenzung der Folgen ausgerichtet, findet also darüber ihre kommunikativen Anschlüsse. ${ }^{8}$

\section{Recht im Katastrophenfall}

Wenn es Aufgabe des Rechts ist, Erwartungssicherheit zu schaffen, auch und gerade dann, wenn sich ansonsten alles verändert, dann liegt ersichtlich ein Spannungsverhältnis darin, dass die Diskontinuität gerade durch Enttäuschung basaler Erwartungen über Sicherheit gekennzeichnet ist. In den älteren Diskussionen darüber, ob denn das Recht den Ausnahmezustand überhaupt regeln könne oder nur auf eine Normallage

5 So hat der gestrandete Holzfrachter »Pallas« vor der deutschen Nordseeküste für die Landesregierung Schleswig-Holstein schwere politische Folgen infolge der Nichtwahrnehmung der symbolischen Dimension des Geschehens gehabt, nicht anders als die Fehleinschätzung der Wahrnehmung der Ölverschmutzung durch die Havarie der »Prestige « für die Regierung Aznar. Die Hochwasserkatastrophe in den neuen Ländern 2002 hat dagegen die Wiederwahlchancen eines Bundeskanzlers durch eine mediale »Retter«inszenierung erhöht.

6 Luhmann, Soziologie des Risikos, 1971, S. 159 f. bestimmt daher aus der ex ante Perspektive von Befürchtungen Katastrophen gerade dadurch, dass die Betroffenen sich von quantitativen Analysen nicht mehr überzeugen lassen; dazu auch Japp, (Fn. 1).

$7 \mathrm{Zu}$ diesem Unterschied Japp, (Fn. 1), S. 80 ff.

8 An der Debatte über den Ausstieg aus der Kernenergie lassen sich der Unterschied zwischen Risikokommunikation und Katastrophenkommunikation deutlich beobachten und einige empirische Anhaltspunkte für die These gewinnen, dass bei letzteren gerade wegen der unbestimmten Kontingenz auf radikale Folgenvermeidung abgestellt wird: Ausstieg aus der Atomenergie; vgl. die Nachzeichnung der Debatte bei Stoll, Sicherheit als Aufgabe von Staat und Gesellschaft, 2003, S. 167 ff. 
bezogen sein könne, kam dies noch zum Ausdruck, ${ }^{9}$ und untergründig schwingt dies noch mit, wenn die Frage gestellt wird, ob denn tatsächlich angesichts der unspezifischen Ungewissheit über Katastrophen rechtliche Regelungen mehr als nur Generalklauseln sein könnten, die der Sache nach die Bewältigung von Katastrophen zur Stunde der Exekutive machen. ${ }^{10}$ Indes, Katastrophen sind nicht notwendig solche des Rechtssystems, wiewohl auch dieses unter Druck geraten kann und nicht selten auch gerät. Aber der Sache nach ist dies nichts Ungewöhnliches, sondern eher eine Frage nach der adäquaten Ausrichtung des Rechtssystems. Nicht nur die Diskussion über die Notstandsverfassung zeigt, dass die Ordnung des Grundgesetzes auf die Bewältigung von Ausnahmesituationen, Notständen und eben auch Katastrophen ausgerichtet ist, also versucht, selbst die Katastrophe rechtsstaatlich zu bewältigen. Oder anders: Selbst für den Fall unbestimmter Kontingenz, der eine Katastrophe ausmacht, versucht das Recht Erwartungsstrukturen zu etablieren, die den Anschluss an die Normallage halten oder zu deren Wiederherstellung dienen sollen. Die Frage ist freilich, ob diese adäquat sind und wieweit diese Form der Restabilisierung reicht und reichen kann.

Im Folgenden geht es nicht um das Risikorecht als Präventionsrecht, sondern um das Recht, das sich mit der Katastrophe aus der Perspektive ihres drohenden Eintritts oder Ablaufs und der Rückkehr zu einem Zustand der Ordnung beschäftigt. Beide hängen allerdings schon deshalb zusammen, weil das Risikorecht gleichsam die primäre Schutzarchitektur vor Katastrophen darstellt, das Katastrophenschutzrecht bei Versagen oder Nichtvorhandensein der einschlägigen Sicherheitsarchitektur zum Einsatz kommt. Dabei ist aus der Perspektive der obigen Diskussion zu beobachten, dass das Katastrophenschutzrecht zunehmend eine Schicht des vorbeugenden Katastrophenschutzes ausbildet, der auf eine effektivere Schadensvermeidung ausgerichtet wird und insoweit wiederum an Risikoanalysen und -bewertungen anknüpft, in denen gleichsam ein re-entry der Unterscheidung von Risiko und Katastrophe stattfindet.

\section{Die Architektur des herkömmlichen Katastrophenschutzrechts}

Katastrophenschutzrecht ist ein Mauerblümchen des juristischen Diskurses. ${ }^{11}$ Fast scheint es so, als hätte die Verbindung mit dem Zivilschutz als gleichsam militärische

9 Als Kronzeuge dafür kann immer noch, wenn auch in anderen Zusammenhängen, Schmitt in Anspruch genommen werden; vgl. ders., Politische Theologie Bd. I, 2. Aufl. 1934, S. 19 f.; ders., Die staatsrechtliche Bedeutung der Notverordnung, in: Verfassungsrechtliche Aufsätze aus den Jahren 1924-1954, S. 235, 257 ff. Zur Diskussion im Rahmen von Art. 91 GG vgl. etwa Volkmann, in: v. Mangoldt/Klein/Starck, GG, Bd. III, Art. 91 Rdn. 5.

10 Auch darin finden sich noch Anklänge an die reine Dezision Carl Schmitts, auf die freilich in den entsprechenden Kontexten nicht mehr verwiesen wird; vgl. etwa Stober/Eisenmenger, Katastrophenverwaltungsrecht - Zur Renaissance eines vernachlässigten Rechtsgebiets, NVwZ 2005, 121, 122.

11 In den Lehr- und Handbüchern des Polizeirechts, wiewohl diesem Bereich traditionell zugeschlagen, finden sich, über einzelne Stichworte hinaus, keine weitergehenden Auseinandersetzungen mit dem Rechtsgebiet mehr. In einzelnen Lehrbüchern findet es sich nicht einmal mehr in den Stichwortverzeichnissen. Daran zeigt sich die Randständigkeit mehr als deutlich; aus neuerer Zeit im Wesentlichen darstellend Stober/Eisenmenger, (Fn. 10), NVwZ 2005, 121 ff., die freilich zu Recht auf die Notwendigkeit einer Auseinandersetzung hinweisen. 
Seite des Katastrophenschutzes dazu beigetragen, dass spätestens mit dem Einfahren der Friedensdividende der 90er Jahre das Thema als erledigt angesehen wurde. ${ }^{12}$ Eine auf Sicherheit ausgerichtete Gesellschaft, die hohe Präventionsanstrengungen unternimmt, mag sich auch das Versagen der Prävention nicht wirklich vorstellen wollen. Angesichts veränderter Szenarien und Bedrohungsanalysen, die zu einem raschen Umbruch des Rechtsgebiets aber auch dessen Wahrnehmung in der Öffentlichkeit führen könnten, dürfte es freilich sinnvoll sein, eine Besichtigung des Verdrängten vorzunehmen. Dies um so mehr, als es doch erstaunlich ist, dass eine Gesellschaft, die jede technisch mögliche Vorkehrung gegen ein Versagen etwa hochtechnischer Systeme einklagt, die Frage nach dem, was passiert und passieren soll, wenn die Prävention versagt, offensichtlich lieber einem öffentlich wenig wahrgenommenen Diskurs von Katastrophenexperten überlässt, gerade so, als handele es sich nicht um hochpolitische Fragen, welche Ressourcen für den Katastrophenschutz zur Verfügung stehen, wie die Überlebenschancen verteilt werden, ob diese gänzlich unter Haushaltsvorbehalt stehen und wer die Entscheidung aufgrund welcher Kriterien trifft, ob Kontaminierte und/ oder schwer Verletzte noch behandelt werden, oder im Interesse anderer aussichtsreicher Kandidaten lieber in betreuter Distanz ihrem prognostiziert unvermeidlichen Ende überlassen werden. Während die rechtsstaatliche Durchformung des Präventionsbereichs immer subtilere Wendungen nimmt, scheint im Katastrophenfall alles rechtsstaatlich ein bisschen derber gestrickt werden zu dürfen. Einleuchtend mag dies denen scheinen, die den Notstand ohnehin für die nichtregelbare Stunde der Exekutive halten. In gewissem Umfang mag das dann plausibel sein, wenn die Katastrophe bereits eingetreten ist. Aber auch davor?

\section{Katastrophen: rechtlich konstruiert}

Katastrophen werden vor allem in den Katastrophenschutzgesetzen der Länder definiert, ${ }^{13}$ und zwar im Wesentlichen durch zwei miteinander verknüpfte Merkmale, die außergewöhnliche Höhe des drohenden oder eingetretenen Schadens und die Unmöglichkeit, diese mit den normalen Mitteln innerhalb der gegebenen Zuständigkeiten zu

12 Dazu und zu der deutlichen Reduktion der Ressourcen für den Zivil- und Katastrophenschutz; vgl. Schöttler, Ist unser Bevölkerungsschutzsystem noch zukunftsfähig, in: Deutsches Komitee für Katastrophenschutzvorsorge e.V. (DKKV), 2000, S. 7 f.; Dombrowsky/ Horenczuk/Streitz, Erstellung eines Schutzdatenatlasses, Zivilschutzforschung Neue Folge Bd. 51, 2003, S. 44.

13 Das Grundgesetz verwendet den Begriff der Katastrophe nicht als solchen, sondern allenfalls mit dem Zusatz der Natur, und auch insoweit schließen sich die einschlägigen Kommentierungen eher an das Katastrophenschutzrecht an, denn umgekehrt. Zugleich spiegelt sich darin freilich auch, dass Katastrophenschutz im Kern eine Länderkompetenz ist. Ansonsten spricht es von Seuchengefahr (Art. 11 Abs. 2, 13 Abs. 7 GG) oder besonders schweren Unglücksfällen (Art. 11 Abs. 2, Art. 35 Abs. 2, 3 GG). Schon der Begriff der Naturkatastrophe ist dabei unscharf geworden; vgl. etwa v. Danwitz, in: v. Mangoldt/Klein/ Starck, GG, Bd. 2 Art. 35 Rdn. 70. Dabei wird außer Acht gelassen, dass diese herbeigeführt werden können, also absichtsvollen oder doch zurechenbaren Entscheidungen unterliegen, das wird dann - etwas gegen den Wortsinn - über die besonders schweren Unglücksfälle einbezogen. 
bewältigen. ${ }^{14}$ Diese doppelte Relationierung der Katastrophe auf Schaden und Fähigkeit der jeweils zuständigen Behörden zur Verhinderung oder Umgang mit den Folgen des Ereignisses hält den Begriff offen und macht zugleich deutlich, dass rechtlich die Annahme einer Katastrophe von dem, was als außergewöhnlich wahrgenommen wird, abhängig ist, also den Kontinuitätserwartungen, und davon, wie die Ressourcen der eigentlich zuständigen Behörden sind. Katastrophen sind also auch rechtlich wahrnehmungs- und zudem ressourcenabhängig und damit von einer Reihe gesellschaftlicher Vorentscheidungen abhängig. Sie sind relativ zum jeweiligen Standard der Katastrophenkultur. ${ }^{15}$ Dabei kommt es folgerichtig auf Ursachen und Zurechnungen nicht mehr an, sondern allein darauf, dass ein katastrophaler Zustand droht oder eingetreten ist. In diesem Sinne ist Katastrophe die rechtliche Konstruktion eines sozialen Ereignisses, das deutlich von der Normalitätserwartung abweicht und eben deshalb außergewöhnliche Maßnahmen und Anstrengungen rechtfertigt.

Die darin liegende Ausblendung der Ursachen und Entscheidungen ist eine Vereinfachung nur solange, wie man die rechtliche Architektur des Umgangs mit Unsicherheit nicht vollständig in die Betrachtung einbezieht. Denn Katastrophenschutzrecht ist nicht primäres Präventionsrecht. Das findet sich in den Sicherheitsanforderungen für großtechnische Systeme, in weiten Teilen des Umweltrechts, in den Vorschriften über Impfungen und die Behandlung von Krankheiten, im sonstigen Recht der polizeilichen Gefahrenabwehr einschließlich der Gefahrenvorsorge und dem Recht der Geheimdienste. Für den Fall des Versagens dieser Primärordnung steht dann das Katastrophenschutzrecht zur Verfügung, als Auffangnetz für die Fälle, in denen die Sicherheitsarchitektur versagt oder aus technologischen Gründen unzureichend ist, weil nach dem Stand des Wissens und der Fertigkeiten keine Verhinderung möglich ist, allenfalls präventive Schadensverringerung, also bei »echten« Naturkatastrophen, wie etwa bei Erdbeben oder Vulkanausbrüchen.

\section{Die drei Säulen des Katastrophenschutzes}

Katastrophenschutz ist, wie die Gefahrenabwehr, als deren Teil sie weithin gilt, grundsätzlich Sache der Länder, obliegt also sowohl ihrer Gesetzgebungs- wie Verwaltungskompetenz. Demgegenüber liegt der Schutz von Bevölkerung und Sach- und Kulturgütern sowie die Beseitigung oder Minderung der Folgen kriegerischer Auseinandersetzungen als Zivilschutz in der ausschließlichen Gesetzgebungskompetenz des Bundes (Art. 73 Nr. 1 GG), der über Art. 87 b Abs. 2 GG auch die Verwaltungskompetenz mit Zustimmung des Bundesrates an sich ziehen kann. Zuständig für den Zivilschutz sind nach dem Zivilschutzgesetz ${ }^{16}$ die für den Katastrophenschutz in den

14 Vgl. für andere Art. 1 Abs. 2 BayKSG; § 2 Abs. 1 BerlKatSG.

15 Dombrowsky/Brauner, Defizite der Katastrophenvorsorge in Industriegesellschaften am Beispiel Deutschlands, Gutachten, Veröffentlichung der Katastrophenforschungsstelle der Christian-Albrechts-Unviersität Kiel, 1996 (http://www.kfs.uni-kiel.de/de_12.php).

16 Vgl. § 2 Zivilschutzgesetz (ZSG) v. 25. März 1997 (BGBl. I S. 726 ff., zuletzt geändert durch Art. 2 des Gesetzes über die Errichtung des Bundesamtes für Bevölkerungsschutz und Katastrophenhilfe v. 27. April 2004 (BGBl. I 2004, S. 630)). 
Ländern zuständigen Behörden, im Regelfall die Landkreise als untere Katastrophenschutzbehörden. ${ }^{17}$ Er wird aber - abgesehen von der durch die nichtrechtsfähige Bundesanstalt Technisches Hilfswerk (THW) gewährten technischen Hilfe und den sonstigen Verwaltungsaufgaben des Bundes, für die das Bundesamt für Bevölkerungsschutz und Katastrophenhilfe zuständig ist ${ }^{18}$ - in Bundesauftragsverwaltung von den Ländern durchgeführt. Die dritte Säule ist dann der militärische Schutz der Bundesrepublik im Falle des äußeren und des inneren Notstandes. ${ }^{19}$

Freilich waren diese drei Säulen auch bisher nur auf den ersten Blick sauber getrennt. Unabhängig davon, dass die Sachverhalte ineinander übergehen können (äußerer Notstand führt zu einem inneren Notstand, die Katastrophe löst inneren Notstand aus), sind die Normen aufeinander bezogen. So können die Kräfte des Zivilschutzes auch für Katastrophenfälle eingesetzt werden, ergänzen die Instrumentarien des Bundes die der Länder und umkehrt. ${ }^{20}$ Ein prägnanter Fall ist hier bekanntlich Art. 35 GG, der über die allgemeine Rechts- und Amtshilfepflicht hinaus den Einsatz nicht nur der Bundespolizei sondern auch anderer Verwaltungseinrichtungen und der Streitkräfte ermöglicht. Allerdings zeigen sich deutlich weitergehende Erosionen der an sich klaren und nur für begrenzte Fallgruppen verschliffenen kompetenziellen und organisatorischen Trennung der Säulen. Das Gesetz zur Neuregelung der Luftsicherheitsaufgaben zeigt dies ebenso an, wie die weiteren Versuche, mit oder ohne Grundgesetzänderung $^{21}$ die Streitkräfte bei terroristischen Bedrohungen im Inland einsetzen zu können.

Auch unabhängig davon ist nicht zu übersehen, dass der Rechtsrahmen des Schutzes vor Katastrophen immer schon weiter zu ziehen war. Das wird deutlich, wenn man sich die Stufen des Umgangs mit Ungewissheit und die Ansatzpunkte ihrer normativen Bewältigung ansieht. Der Bereich der eigentlichen Prävention, also der Bereich des Umwelt-, technischen Sicherheitsrechts, des Rechts der Geheimdienste und der Gefahrenabwehr der Polizei- und Ordnungsbehörden, sowie der Planung räumlicher und fachlicher Natur der bestimmten Schadensverläufe wird zunehmend auch für Zwecke des vorbeugenden Katastrophenschutzes im Sinne einer präventiven Schadensminderung genutzt. Deutlich ist dies an dem jüngst in Kraft getretenen Gesetz zur Verbesserung des vorbeugenden Hochwasserschutzes, ${ }^{22}$ das Änderungen des Wasser-

17 Ausführlich Stober/Eisenmenger, (Fn. 10), NVwZ 2005, 121, 124 ff.

$18 \mathrm{Vgl}$. Gesetz über die Errichtung des Bundesamtes für Bevölkerungsschutz und Katastrophenhilfe v. 27. April 2004 (BGB1. I 2004, S. 630).

19 Für den Fall des äußeren Notstandes hat der Gesetzgeber in den sogenannten Sicherstellungsgesetzen eine Reihe von Vorkehrungen getroffen, die allerdings auf den Fall des Art. 80 a GG bezogen sind, wie etwa das Arbeitssicherstellungsgesetz, das Bundesleistungsgesetz, das Ernährungssicherstellungsgesetz, das Verkehrssicherstellungsgesetz und das Wirtschaftssicherstellungsgesetz. Andere, wie das Post- und Telekommunikationssicherstellungsgesetz sind insoweit weiter und beziehen auch Naturkatastrophen und Unglücksfälle ein.

20 Vgl. etwa $§ 6$ Abs. 2, 10 Abs. 2, 11 ZSG.

21 Zur Notwendigkeit einer Grundgesetzänderung Soria, Polizeiliche Verwendungen der Streitkräfte, DVBl. 2004, S. 597 ff.; Stober/Eisenmenger, (Fn. 10), NVwZ 2005, 121, 123 m.w. N.

22 Gesetz zur Verbesserung des vorbeugenden Hochwasserschutzes v. 31. Mai 2005 (BGBl. I S. 1224). 
haushaltsgesetzes, des Bauplanungsrechts und der Raumplanung bringt, die auf einen vorbeugenden Katastrophenschutz ausgerichtet sind. Im Bereich des Katastrophenpräventionsrechts liegen insoweit eine Vielzahl verschränkter Zuständigkeiten der verschiedenen Ebenen und Säulen vor, gleichsam der Normalfall einer bundesstaatlichen Ordnung. Das zeigt sich im Bereich der Infektionskrankheiten, wo dem Bund die Gesetzgebungskompetenz zusteht und er - etwa mit dem Robert Koch-Institut - partielle Verwaltungskompetenzen hat, die im Übrigen aber bei den Ländern liegen. Hinzu tritt etwa das Arneimittel- und Medizinprodukterecht, einschließlich der europäischen Dimensionen, die ebenfalls vorbeugend auf den Katastrophenfall ausgerichtet werden. ${ }^{23}$ Nichts anderes gilt etwa bei den großtechnischen Systemen, wie den Infrastrukturen Eisenbahn, Luftverkehr, gefährliche Anlagen etc. Insoweit offenbart sich zumal das Recht des (vorbeugenden) Katastrophenschutzes als ein relativ komplexes Gebilde, das schon zu überblicken schwierig ist, dessen Ausrichtung auf ein bestimmtes Ziel freilich besondere Herausforderungen stellt. Katastrophenschutzrecht ist unter Einbeziehung des vorbeugenden Katastrophenschutzes insoweit eine Querschnittsmaterie. Während im konkreten Fall im Wesentlichen eine Zuständigkeitskonzentration bei den Katastrophenschutzbehörden eintritt, also das eigentliche Problem in der Integration ganz unterschiedlicher Akteure in einen konkreten Handlungszusammenhang besteht, hat man es im vorbeugenden Katastrophenschutz mit einer Vielzahl von Kompetenzen und Organisationen zu tun, die im Vorfeld koordiniert werden müssen.

\section{Das herkömmliche Katastrophenschutzrecht}

Das herkömmliche Katastrophenschutzrecht, wie es sich in den Katastrophenschutzgesetzen der Länder findet, ist auf den ersten Blick wesentlich Organisationsrecht, nicht materielles Recht, nicht Verfahrensrecht, wiewohl naturgemäß Eingriffsvoraussetzungen und Instrumente gegenüber dem Bürger in der Katastrophensituation normiert werden. Das ist aus der Tradition des Rechtsgebiets her einleuchtend. Es dient ja gerade dazu, eine Grenzsituation zu bewältigen, der die zuständigen Behörden mit ihren Mitteln nicht gerecht werden können. Dies geschieht im Wesentlichen durch das Zusammenlegen der Ressourcen und deren Ausrichtung auf die jeweilige Situation. Insoweit ist es wesentlich das Recht der Integration einer fachlich spezialisierten, differenzierten und in verschiedene Ebenen gegliederten Verwaltung in einen temporären, ereignisabhängigen Verwaltungsverbund und dessen Unterstellung unter eine einheitliche Führungsorganisation - einschließlich der Einrichtungen des Bundes, wie der Bundeswehr in den Fällen des Art. 35 Abs. 2 und - strittig - Abs. 3 GG, des Bundesgrenzschutzes und des Technischen Hilfswerkes. Es ist daher in wesentlichen Teilen Innenrecht der Verwaltung, was sich nicht zuletzt anhand der Vielzahl der Verwaltungsvorschriften zeigt, die jenseits der Katastrophenschutzgesetze und den dazu ergangenen Rechtsverordnungen die eigentliche Tätigkeit der Katastrophenschutzbehörden regeln. Modern war es immer schon darin, dass diese Integration gesellschaftliche

23 Vgl. dazu etwa Nationaler Influenza-Pandemieplan Teil II. Analysen und Konzepte für Deutschland (Robert Koch-Institut, 2005), S. 31 hinsichtlich der Zulassungsverfahren für Impfstoffe. 
Kräfte umfasste, die privaten Hilfsorganisationen und private ehrenamtliche Helfer, die freilich anders als in modernen Erscheinungsformen der Zusammenarbeit von Staat und Privaten in die Führungsorganisation integriert werden. ${ }^{24}$ Es begründet aus sonstigen Rechtsgebieten nicht bekannte Hilfeleistungspflichten der Bürger und Mitwirkungspflichten all derjenigen, die einen Beitrag zur Katastrophenbewältigung leisten können..$^{25}$

\section{a) Vorbeugung und Planung}

Selbstverständlich enthielt dieser Ordnungsrahmen immer schon Elemente der Planung, allerdings eher im Sinne der Abstimmung und Vorhaltung der nötigen Ressourcen. Dieser war - dem Ziel des Katastrophen- und Zivilschutzes entsprechend - daran ausgerichtet, einen bestimmten Standard an Ressourcen flächendeckend vorzuhalten. Ebenso kannte dieser Ordnungsrahmen immer schon Elemente der Vorbeugung, allerdings weniger im Sinne der Katastrophenprävention, sondern vor allem, um das Zusammenwirken der verschiedenen Einrichtungen zu proben und damit deren Funktionsfähigkeit im Ausnahmefall sicherzustellen, für den sich im Alltag keine Routinen einstellen. Es fällt freilich auf, dass eine Verbindung der Ebenen von Prävention und (vorbeugendem) Katastrophenschutz nicht wirklich geleistet wurde und wird. So zeigt etwa die Analyse der Hochwasservorsorge in Deutschland im Nachgang zu der Flutkatastrophe an der Elbe 2002 erhebliche Defizite nicht nur auf der Ebene des Katastrophenschutzes im engeren Sinne auf, ${ }^{26}$ sondern eben auch solche der Vorbeugung, ${ }^{27}$ von der integralen Raumplanung über die Wasserbewirtschaftung bis hin zum Bauschutz. Exemplarisch zeigt sich hier, dass die Ebenen der eigentlichen Prävention (Deichbau), des vorbeugenden Katastrophenschutzes (Raumplanung und Bauschutz) und Katastrophenabwehr aufeinander bezogen sind, aber normativ wie praktisch nicht hinreichend aufeinander bezogen werden. Erst durch die jüngste Gesetzgebung des Bundes ist hier eine Änderung hinsichtlich des Hochwasserschutzes eingetreten. ${ }^{28}$

\section{b) Prävention, Vorbeugung und Abwehr}

Damit zeichnet sich eine Architektur ab, die (auf den ersten Blick) zweistufig ist: das primäre Präventionsrecht, also insbesondere das Risikoverwaltungs- und technische Sicherheitsrecht, und das sekundäre Katastrophenschutzrecht, gleichsam als Auffangordnung für das Versagen der primären Vorkehrungen. Allerdings ist Letzteres ebenfalls zweistufig, umfasst also diejenigen Vorkehrungen, die auf die Vorbeugung gerichtet sind, also die Vorbereitung auf den Katastrophenfall und das Recht des Katastrophenfalles, das dazu dient, den operativen Umgang mit der drohenden oder eingetretenen konkreten Ka-

24 Vgl. §§ 40 f. SächsBRKG.

25 Vgl. etwa $\S \$ 54,55,57$ SächsBRKG.

26 Dazu Bericht der unabhängigen Kommission der Sächsischen Staatsregierung Flutkatastrophe 2002.

27 Ausführlich dazu Deutsches Komitee für Katastrophenvorsorge e.V. (DKKV), Hochwasservorsorge in Deutschland. Lernen aus der Katastrophe 2002 im Elbegebiet, 2003.

28 Gesetz zur Verbesserung des vorbeugenden Hochwasserschutzes v. 31. Mai 2005 (BGBl. I S. 1224). 
tastrophe zu regeln. Primäres Präventionsrecht wie sekundäres Katastrophenschutzrecht sind der Sache nach aufeinander bezogen, wiewohl dies im juristischen Diskurs selten thematisiert wird. Analytisch gesehen stellt der vorbeugende Katastrophenschutz - jedenfalls dort, wo es nicht um reine Naturkatastrophen geht - die Verbindung zwischen beiden Teilen her. Vorbeugender Katastrophenschutz knüpft an die Risikoanalysen des primären Sicherheitsrechts an und richtet mit je eigenen, auf den Versagensfall und die dann notwendigen Schaden minimierenden Vorkehrungen und dazu nötigen Ressourcen, das operative Katastrophenschutzrecht aus, wie zunehmend deutlich wird, aber rechtlich bisher wenig ausgeformt und rechtswissenschaftlich bisher wenig strukturiert worden ist. In diesem Sinne lassen sich primäres Sicherheitsrecht und sekundäres Katastrophenschutzrecht als Teil einer einheitlichen Sicherheitsarchitektur verstehen.

\section{c) Die Inkorporation von Expertise}

Katastrophenschutz ist wesentlich auf Expertise angewiesen, etwa technische Expertise, Expertise im Management komplexer Situationen, medizinische Expertise. Sie wird integriert über fachlich spezialisierte Organisationen privater oder öffentlicher Natur und über mehr oder weniger dauerhafte Netzwerke, als deren Knotenpunkte die früh auf Initiative von Heisenberg gegründete Schutzkommission des Bundes sowie die Akademie für Krisenmanagement, Notfallplanung und Zivilschutz des Bundes sowie Foren, Arbeitsgemeinschaften und Ständige Konferenzen weitgehend die Arbeit der Standardisierung, der Beobachtung und Evaluation leisten, ohne dass dies notwendigerweise politisch und rechtlich rückgebunden wäre.

\section{d) Katastrophenschutzrecht und Öffentlichkeit}

Die Öffentlichkeit oder der Bürger, sofern nicht über die Hilfsorganisationen integriert oder für die Katastrophenbewältigung in Dienst genommen, kommt in diesem Rahmen eher als Adressat staatlicher Katastrophenbewältigungsleistungen, also als Opfer und als passives Mitglied einer über Selbstschutz und Selbsthilfe aufzuklärenden, ansonsten aber - notfalls durch Platzverweise - aus dem Geschehen herauszuhaltenden Öffentlichkeit vor. Erst neuerdings wird etwa die Integration der freiwilligen Katastrophenhelfer angemahnt. ${ }^{29}$ Wie Fremdkörper nehmen sich daher auch die Vorschriften über die Auslegung und Öffentlichkeitsbeteilung von externen Notfallplänen für schwere Unfälle mit gefährlichen Stoffen aus, ${ }^{30}$ die nicht auf einen Paradigmenwechsel im Katastrophenschutz hindeuten, sondern eher auf das Einsickern europäischer Öffentlichkeitskonzeptionen in das deutsche Katastrophenschutzrecht. ${ }^{31}$ Ohne eine Einbeziehung der Bevölkerung und Nutzung ihrer Selbstschutz- und Selbsthilfepotentiale kann allerdings eine Katastrophe nur schwer angemessen bewältigt werden. ${ }^{32}$

29 Rechenbach, Möglichkeiten zur Steigerung der Abwehrbereitschaft des Katastrophenschutzes, Zivilschutzforschung Neue Folge Bd. 55, 2004, S. 141, 173.

30 Vgl. statt vieler § 43 SächsBRKG.

31 Sie stellen eine Umsetzung der RL 96/82/EG (Abl. EG 1997 Nr. L 10 S. 13) dar.

32 Dazu nochmals Bundesverwaltungsamt, Zentralstelle für Zivilschutz, Neue Strategien zum Schutze der Bevölkerung, 2004, Schriftenreihe: Wissenschaftsforum Bd. 4, S. 29 ff. 


\section{Neue Anforderungen an den Katastrophenschutz}

Unübersehbar hat sich die Situation des Katastrophenschutzes verändert. Das DreiSäulen Modell hatte seine (auch verfassungsrechtliche) Grundlage in der Dichotomie von »Friedens «katastrophen und Zivilschutz im Verteidigungsfall. Letzterer hatte relativ klar überschaubare Konturen, die Szenarien waren bekannt, die Vorlaufzeit möglicher Einsätze relativ lang, die Ressourcen und Einsätze planbar, da man unterstellen konnte, dass man es mit relativ rational kalkulierenden Akteuren zu tun hatte. Flächendeckender Schutz der Zivilbevölkerung im Verteidigungsfall war das Ziel, militärisch geprägt in Anlage und Durchführung. Die »Friedens«katastrophen hatten naturgemäß eine andere Anlage, erforderten aber nicht notwendig andere Strukturen, da die Ressourcen ohnehin wechselseitig nutzbar sein mussten und zudem hinreichend lange Vorwarnzeiten angenommen wurden, die von Fall zu Fall eine Aufstockung der Einsatzkräfte ermöglichen sollten. ${ }^{33}$ Diese Tradition prägt die Regelungsansätze bis in die verfassungsrechtliche Kompetenzordnung hinein.

Diese duale Struktur, insbesondere aber die mit ihr verbundenen Leitbilder des flächendeckenden Zivilschutzes gegenüber Bodenkampfhandlungen und andererseits singulären Naturereignissen oder Versagen von großtechnischen Systemen, ist infolge einer Reihe von Veränderungen, wenn nicht obsolet geworden, so doch nachhaltig verändert worden. ${ }^{34}$ An die Stelle der bipolaren Weltordnung mit den daran anknüpfenden Szenarien der Bedrohung sind diffuse Bedrohungen durch terroristische Anschläge getreten, die in ihrem Eintritt ungewiss und in ihrem Ablauf kaum planbar, in ihrer Intensität aber auf maximale Effekte im Sinne der Angsterzeugung oder Zerstörung von Infrastruktur und Tötung von Menschen gerichtet sind. Darüber hinaus sind die früheren, eher seltenen Naturereignisse in ihrem Eintritt häufiger und die Rahmenbedingungen instabiler geworden. Infolge des Klimawandels sind überraschende Schadensereignisse deutlich gesteigerten Ausmaßes zu erwarten. Wirksame Katastrophenbekämpfung setzt zudem nicht nur im Einzelfall Hilfe in anderen Ländern voraus. Die Unterstützung ist nicht selten erforderlich, um im eigenen Land Katastrophen zu verhindern, wie etwa an der Verhinderung von Pandemien leicht gezeigt werden kann. ${ }^{35}$ Hinzu kommen veränderte staatliche und gesellschaftliche Rahmenbedingungen. Dazu rechnet etwa der Rückzug der Bundeswehr aus der Fläche, die die gleichsam »stille Reserve« des Katastrophenschutzes ausmachte. Die Übernahme internationaler Kriseneinsätze führt dazu, dass wichtige Ressourcen im nationalen Katastrophenfall unverfügbar sein können. Die Modernisierung des öffentlichen Sektors einschließlich der Privatisierungen führt dazu, dass die bisher selbstverständlichen Zugriffe auf deren Ressourcen heute nicht mehr unterstellt sondern eher hergestellt wer-

33 Rechenbach, (Fn. 29), S. 144.

34 Ausführlich dazu Bundesverwaltungsamt (Hrsg.), Zweiter Gefahrenbericht der Schutzkommission beim Bundesminister des Inneren. Bericht über mögliche Gefahren für die Bevölkerung bei Großkatastrophen und im Verteidigungsfall, 2001.

35 Vgl. Stollorz, Lokal Handeln, global stoppen, FASZ v. 7. Aug. 2005, S. 56. 
den müssten. ${ }^{36}$ Der Massentourismus erhöht zweifelsohne die Gefahren für Epidemien mit seltenen Erregern, für die die notwendigen Mittel nicht notwendig vorhanden oder verfügbar sind. Alles in allem wird man sagen können, dass der Katastrophenschutz, per se bezogen auf eine Situation, in der die primären Schutzinstrumente der Gesellschaft versagen, es mit schwerer prognostizierbaren Schadensverläufen in einer deutlich komplexeren Umwelt zu tun hat. Entsprechend müssen Instrumente flexibler und dynamischer werden; strategische Szenarien, Methoden der Simulation von Problemlagen treten an die Stelle von Erfahrungen. Angesichts der Heterogenität und Verflochtenheit der Schadensverläufe gewinnt das Management von Ressourcen und Netzwerken an Bedeutung. ${ }^{37}$ Die alten Abgrenzungen der Säulen und die Segmentierung einzelner Problembereiche werden damit brüchig.

\section{Vorbeugung: Von der flächendeckenden Gleichheit zum risikodifferenzierten Schutzniveau}

Insoweit fehlen ersichtlich anspruchsvollere Planungen und Strukturen, die auch für auBergewöhnliche Gefahren- und Schadenslagen und für solche unerwarteten und in ihrem Ablauf schon aufgrund mangelnder Erfahrungen schwer strukturierbaren Lagen, wie sie etwa durch terroristische Anschläge auf Infrastrukturen, das Freisetzen von ABC-Potentialen und ihre Kombination eintreten können. ${ }^{38}$ Sie lassen sich schon aufgrund ihrer geringen oder gar nicht vorhandenen Vorlaufzeit, des Nichtwissens über mögliche Orte und Verläufe und des potentiell hohen Anfalls von Verletzten und Getöteten nur schwer strukturieren und verlangen daher wesentlich flexiblere Strukturen, zugleich aber auch differenzierte, an möglichen Bedrohungsszenarien ausgerichtete Strukturen. Angemessene Ressourcen lassen sich zu angemessenen Kosten nicht flächendeckend vorhalten, sondern verlangen nach Risikopotentialen differenzierte und flexibel einsetzbare Instrumente. ${ }^{39}$ Das erhöht den Koordinationsaufwand der Akteure, verlangt andere Allokationsmechanismen der Verteilung knapper Ressourcen und erhöht die Anforderungen an die Verlässlichkeit des Gesamtssystems des Katastrophenschutzes. Dies erfordert eine veränderte Struktur, deren Grundlage Risikoanalysen und -bewertungen sein müssten. ${ }^{40}$ Ein modernes Risikomanagement, wie es auf der Präventionsebene selbstverständlich

36 Ein Beispiel für viele wäre die Privatisierung von Krankenhäusern, aber auch deren Handeln unter finanziellen Restriktionen und Wettbewerbsbedingungen, die es keineswegs als sicher erscheinen lassen, dass bei einem Massenanfall von Verletzten hinreichend Behandlungskapazitäten zeitgerecht zur Verfügung stehen. Die Veränderung der gesellschaftlichen Produktionsweise - etwa just in time Produktion - lässt es nicht zu, auf Vorräte der Apotheken zu vertrauen, noch kann man darauf setzen, dass Medikamente in hinreichendem Umfang überhaupt in time produziert sind.

37 Ausführlich dazu Bundesverwaltungsamt, Zentralstelle für Zivilschutz, Neue Strategien zum Schutze der Bevölkerung, 2004, Schriftenreihe: Wissenschaftsforum Bd. 4.

38 Eine ausführliche Analyse findet sich im Zweiten Gefahrenbericht der Schutzkommission beim Bundesminister des Inneren, Zivilschutzforschung Neue Folge 48, 2001.

39 Rechenbach, (Fn. 29).

40 Ansätze dazu finden sich in der Gefährdungsanalyse das Landes Hessen aus dem Jahre 2000, die freilich auch die Schwierigkeiten deutlich macht, weil einheitliche Standards einer solchen Risikoanalyse im Bundesstaat derzeit fehlen. 
geworden ist, würde den Katastrophenschutz als Teil einer Sicherheitsarchitektur verstehen müssen. Insoweit bedürfte es der Verzahnung von Risikoanalysen der Präventionsebene mit denen der Katastrophenplanung, die gerade auf Versagen des Primärschutzes bezogen ist. Ein wirksames Risikomanagement bedürfte dann freilich auch der Festlegung von Schutzzielen, Interventionszielen und Leistungsprofilen, ${ }^{41}$ die dann für die einzelnen Einrichtungen auszubuchstabieren und aufeinander zu beziehen wären. Dies führt schon angesichts knapper Ressourcen zur sachlichen Ausdifferenzierung von Schutzstandards und Versorgungsstufen zwischen flächendeckender Vorhaltung eines Grundschutzniveaus und dessen Erhöhung für spezielle Schadensverläufe und zugleich zu einer räumlichen Differenzierung des Katastrophenschutzes. ${ }^{42}$

Diese zu strukturieren wäre Aufgabe eines angemessenen normativen Rahmens. Risikoanalysen und -bewertungen finden als rechtlich strukturierte Verfahren im Katastrophenschutz kaum eine Anwendung, allenfalls behördenintern, auch wenn die Katastrophenschutzgesetze über die Alarm- und Einsatzpläne hinaus z.T. die Untersuchung von Katastrophenschutzgefahren und die Erstellung und Fortschreibung von Katastrophenschutzplänen vorsehen. ${ }^{43}$ Deren Standards, Schutzziele und Schutzniveaus sind allerdings nicht ersichtlich, schon gar nicht länderübergreifend harmonisiert. Allenfalls Ansätze finden sich in den neueren Katastrophenschutzgesetzen. ${ }^{44}$ Eine Verzahnung der Ebenen von Prävention, vorbeugendem und abwehrendem Katastrophenschutz ist derzeit nicht erkennbar. ${ }^{45}$

41 Dazu allgemein jüngst noch einmal Böhm, Risikoregulierung und Risikokommunikation als interdisziplinäres Problem, NVwZ 2005, 609, 613 m.w.N.

42 Vgl. dazu Bundesverwaltungsamt, Zentralstelle für Zivilschutz, Neue Strategien zum Schutze der Bevölkerung, 2004, Schriftenreihe: Wissenschaftsforum Bd. 4, S. 42 ff.; Rechenbach, (Fn. 29), S. 174. Insoweit wird zwischen vier Versorgungsstufen unterschieden: dem normierten alltäglichen Schutz, dem standardisierten flächendeckenden Grundschutz, dem erhöhten Schutz für gefährdete Regionen und Einrichtungen und dem Sonderschutz mit Hilfe von Spezialkräften für von Bund und Ländern gemeinsam definierte Gefahren.

43 Vgl. etwa §§ 7, 10 Nds. KatSG; § 6 LKatSG Schleswig-Holstein; § 29 HBKG.

44 In Ansätzen anders ist dies im Bereich des Rettungsdienstes, bei dem sich planerische Instrumente (Landesrettungsdienstpläne als Rahmenplanung und Bereichspläne für die einzelnen Rettungsdienstbereiche) finden, sowie Vorschriften über die zu erwartenden und vertraglich zu vereinbarenden Leistungsstandards bei der Übertragung der Aufgabe an private Leistungserbringer; vgl. $\S 26$ ff. SächsBRKG. Hier schlagen sich Formen aus dem Sozialrecht nieder, was angesichts der Notwendigkeit der Regelung der Kostentragung durch die Krankenkassen nicht verwundert. Im eigentlichen Katastrophenschutzrecht finden sich in den neueren Gesetzen im Rahmen der Katastrophenvorbeugung Untersuchungspflichten über drohende Katastrophengefahren und die Aufstellung allgemeiner Katastrophenschutzpläne; vgl. etwa § 36 Abs. 1 Nr. 2, 5 SächsBRKG und die Verpflichtung anderer Träger zur Erstellung von Einsatz- und Alarmplänen; vgl. § 56 SächsBRKG; diese sind freilich Teil des Innenrechts und werden - anders als die Notfallpläne - nicht einem Verfahren der Öffentlichkeitsbeteiligung unterworfen. Insofern lässt sich auch nicht sicher sagen, dass diese Pläne tatsächlich bestehen und aktualisiert werden. Nimmt man die Angaben in der Literatur zu den Alarmplänen der Krankenhäuser als Gradmesser, ist Optimismus kaum angebracht.

45 Dazu Deutsches Komitee für Katastrophenvorsorge e.V. (DKKV), Hochwasservorsorge in Deutschland. Lernen aus der Katastrophe 2002 im Elbegebiet, 2003. 


\section{Katastrophenabwehr: Situatives Management eines hochdifferenzierten Systems}

Eine solchermaßen differenzierte Katastrophenschutzarchitektur verlangt freilich mehr noch als bisher die horizontale und vertikale Abstimmung der einbezogenen Einrichtungen. Diese freilich läuft nicht nur an den föderalen Strukturen auf, sondern hat es auch mit Abstimmungs- und Koordinationsproblemen zwischen den unterschiedlichen Trägern des Katastrophenschutzes zu tun, ${ }^{46}$ die sich noch potenzieren, wenn sich länderübergreifende Koordinations- und Abstimmungsnotwendigkeiten ergeben. ${ }^{47}$

Ungeachtet der immer wieder betonten Vorbildlichkeit des deutschen Systems des Katastrophenschutzes ergeben sich schon aus der Sache heraus nahe liegende Probleme, die sich denn auch in wissenschaftlichen Rekonstruktionen von Katastropheneinsätzen deutlich zeigen. ${ }^{48} \mathrm{Im}$ Ausgangspunkt muss man annehmen, dass sich das System Katastrophenschutz erst im Einsatzfall lagespezifisch bilden muss, also sich selbst situativ organisieren muss, wenn auch entlang mehr oder weniger eingeübter Pfade. Ein solches System braucht Zeit und dies umso mehr, je weniger Routinen sich ausgebildet haben. Deshalb bildet sich in der unmittelbaren Situation denn auch eher ein System von unten durch den unmittelbaren Zugriff derjenigen, die zuerst einsatzfähig sind, das über informelle Netzwerke seine Ressourcen rekrutiert und damit incremental handelt. Dieser verlängert sich, je weniger die Strukturen abgestimmt sind und je mehr die jeweiligen Akteure ihrer Organisationslogik folgen. Und er verlängert sich, je mehr strukturelle Barrieren zwischen den verschiedenen Einsatzkräften bestehen, sei es durch Informations- und Kommunikationsbarrieren, sei es durch nicht aufeinander abgestimmte Strukturen, von den Führungsstrukturen ${ }^{49}$ bis hin zu technischen Inkompatibilitäten, wie die viel beklagte Tatsache, dass die Katastrophenschutzkräfte kein integriertes Kommunikationsnetz besitzen. Insoweit ist nicht zu übersehen, dass der Katastrophenschutz, die Analysen ex post zeigen es immer wieder, nach wie vor an Koordinations- und Kooperationsproblemen, an dem Fehlen einheitlicher Standards und an einer mangelnden Professionalität der Katastrophenschutzbehörden leidet.

\section{Verfassungsrechtliche Anfragen}

Das führt zu verfassungsrechtlichen Anfragen an den Katastrophenschutz, die hier freilich nur exemplarisch aufgenommen werden können.

46 Als Beispiel für viele kann die Einbeziehung der Stellen des Öffentlichen Gesundheitsschutzes genannt werden. Dazu etwa Pfenninger/Himmelseher/König, Untersuchung zur Einbindung des Öffentlichen Gesundheitsdienstes in die katastrophenmedizinische Versorgung in der Bundesrepublik Deutschland, Zivilschutzforschung Neue Folge Bd. 54, 2004; auch Obladen, Rettungsdienst und Katastrophenschutz aus Public Health-Perspektive. Ansatzpunkte für den ÖGD, Landesinstitut für den Öffentlichen Gesundheitsdienst, NRW, 2001.

47 Vgl. die zusammenfassende Schwachstellenanalyse bei Rechenbach, (Fn. 29), S. 160 ff.

48 Vgl. Streitz/Dombrowsky, Die Analyse der Katastrophenabwehr als Netzwerk und als Kommunikation, in: Deutsches Komitee für Katastrophenvorsorge e.V. (DKKV), Hochwasservorsorge in Deutschland. Lernen aus der Katastrophe 2002 im Elbegebiet, 2003, S. 100 ff.

49 Zum Ganzen vgl. Arbeitsgemeinschaft der Leiter der Berufsfeuerwehren (AGBF), Führung und Leitung im Katastrophenschutz in der Bundesrepublik Deutschland, Thesen v. 23. 06. 2005. 


\section{Katastrophenschutz im Bundesstaat}

Der Katastrophenschutz ist zumal als vorbeugender eine Querschnittsmaterie. Schon dies lässt Abstimmungsprobleme zwischen Ressorts und Ebene im Bundesstaat erwarten. Hinzu kommt die oben schon dargestellte Veränderung der Anforderungen. Schon immer gab es für den operativen Katastrophenschutz - wie der Sache nach noch jede nachträgliche Schadenanalyse bestätigt hat - Abstimmungsprobleme, die auch etwas mit der bundesstaatlichen Kompetenzaufteilung zu tun hatten, die aber angesichts der Veränderungen der Anforderungen an den (vorbeugenden) Katastrophenschutz noch einmal zugenommen haben. Dies gilt umso mehr, als ein risikodifferenzierter Katastrophenschutz vor allem gemeinsame Standards voraussetzt, ${ }^{50}$ um überhaupt einheitlich Risikopotentiale bewerten zu können und dementsprechend die Ressourcen allozieren zu können. Die sich hier ergebenden Schwierigkeiten werden verstärkt dadurch, dass keine Gesetzgebungskompetenz den Bund in die Lage versetzt, einheitlich Standards vorzugeben. So bleibt es bei der Koordinierung im Kooperationsgeflecht von Bund und Ländern, mit den bekannten Problemen der nur negativen Koordination und der Intransparenz und Diffusion von Verantwortlichkeit, die noch einmal durch das Geflecht informeller Netzwerke von staatlichen und gesellschaftlichen Akteuren gesteigert wird. Die im Katastrophenschutz immer wieder zu findende Metapher von der Gemeinschaftsaufgabe Katastrophenschutz ${ }^{51}$ kann daher nicht über das Fehlen einer solchen im Grundgesetz hinwegtäuschen, was Ausweichlösungen der informellen Koordination und Kooperation begünstigt. Die Kosten der Intransparenz sind freilich beachtlich, weil es schon schwer fällt, den Stand des vorbeugenden Katastrophenschutzes überhaupt zu erheben und die Vielzahl von informellen Foren und Netzwerken überhaupt normativ zu rekonstruieren. Zwar ist von Seiten des Bundes im Rahmen der einstweilen gescheiterten Föderalismusreform 2004 auf eine Veränderung der grundgesetzlichen Kompetenzen gedrungen worden, indes ohne Ergebnis. Das begünstigt Forderungen nach einer Grundgesetzänderung nach Maßgabe von wirklichen oder vermeintlichen Bedrohungsszenarien, wie die nach dem Einsatz der Bundeswehr im Inneren, die ihren Grund in der Erschöpfung der Ressourcen der Polizeien der Länder, der Verschleifung von innerer und äußerer Sicherheit und den veränderten Aufgaben der Bundeswehr bei Auslandseinsätzen finden soll. Vom Blickwinkel des Katastrophenschutzes ist dies freilich eher ein Randthema, das von den eigentlichen Problemen abzulenken geeignet ist, ${ }^{52}$ die eben nicht zuletzt Ressourcenfragen, vor allem aber

$50 \mathrm{Zu}$ den Problemen in Folge einer fehlenden Standardisierung Rechenbach, (Fn. 29), S. 159 ff.

51 Rechenbach, (Fn. 29), S. 160.

52 Einmal abgesehen davon, dass es aus föderaler Sicht ein wenig überzeugendes Argument der ansonsten auf ihre Eigenstaatlichkeit pochenden Länder ist, auf die Erschöpfung der Ressourcen der Polizeien der Länder zu verweisen. Diese angemessen auszustatten, gehört zu ihren Kernverantwortlichkeiten, deren Nichtwahrnehmung keinen Grund dafür abgibt, die über lange Zeit bewährte Trennung von militärischen und zivilen Verantwortlichkeiten aufzulösen. 
auch Abstimmungs- und Verteilungsfragen sind, die mit einem wie auch immer begrenzten Einsatz der Bundeswehr nicht beantwortet sind.

Die Abstimmung der Kompetenzen vor dem Hintergrund veralteter Leitbilder zeigt sich exemplarisch im Rahmen von Art. 35 Abs. 2, 3 GG. Diese in den Kontext der Amts- und Rechtshilfe gestellten Vorschriften warfen schon immer Schwierigkeiten eines angemessenen Verständnisses des Zusammenwirkens von Bund und Ländern auf. Dies zumal dann, wenn es um einen Fall der überregionalen Katastrophenhilfe im Rahmen von Art. 35 Abs. 3 GG ging. Die Einrichtungen des Bundes, die Bundespolizei und die Streitkräfte sollen entweder - wie insbesondere im Falle der regionalen Katastrophenhilfe des Art. 35 Abs. 2 GG - im Rahmen der Führungsorganisation der $\mathrm{zu}$ unterstützenden Länder handeln, ${ }^{53}$ oder aber zwar vom Bund eingesetzt werden, wie es der Text des Art. 35 Abs. 3 GG denn auch nahe legt, aber an das materielle Recht der Länder beim Einsatz gebunden sein, also bei per definitionem länderübergreifendem Einsatz an das Recht eines jeden zu unterstützenden Landes gebunden sein. ${ }^{54}$ Das kann nicht gut damit gerechtfertigt werden, dass es hier um Unterstützung der Länder in ihrem Bereich geht, wo es der Sache nach gerade um länderübergreifende Belange geht. Hier dürfte die Annahme eines Kooperationsverhältnisses jedenfalls für den Fall des Art. 35 Abs. 3 GG näher liegen, das dann freilich auch gesetzlich ausgestaltet werden müsste und zwar von beiden Ebenen aus.

Ungeachtet dieser schon schwierigen und im Einsatzfall pragmatisch camouflierten Probleme fällt freilich ins Gewicht, dass Art. 35 Abs. 2, 3 GG vor dem Leitbild von Katastrophen als punktuelle Ereignisse interpretiert werden. ${ }^{55}$ Damit wird die vorbeugende Dimension nicht zureichend berücksichtigt, also die einer Kooperation und Abstimmung mit den übrigen katastrophenschutzrelevanten Akteuren. Das verlangt Informationsaustausch, Gefährdungsanalysen, Planung der Ressourcen und deren zeitgerechte Dislozierung, für die der punktuelle Einsatzfall dann in der Tat das Ereig-

53 So etwa statt vieler Bauer, in: Dreier (Hrsg.), GGK I Art. 35 Rdn. 25, 27, der dies einheitlich für die regionale und überregionale Katastrophenhilfe annimmt. Das stellte auch im Rahmen der regionalen Katastrophenhilfe immer schon in gewissem Umfang eine Fiktion dar, weil die Führungs- und Managementkompetenzen der Bundespolizei und der Streitkräfte jenen der zivilen Katastrophenschutzbehörden schon immer so überlegen waren, dass es billigend zu einer weit über die Unterstützung hinausreichenden Übernahme von Führungsverantwortung kam und kommt.

54 So etwa von Danwitz, in: v. Mangoldt/Klein/Stark, GG Bd. 2, Art. 35 Rdn. 80 ff., der allerdings annimmt, dass die Bundeseinsatzkräfte weiterhin den Weisungen des Bundes unterstehen, diese aber im Einvernehmen mit den beteiligten Ländern ausgeübt werden müssten. Vgl. auch Erbguth, in: Sachs (Hrsg.), GG, Art. 35 Rdn. 41. Den dadurch offensichtlich werdenden Problemen kann man nur entgehen, wenn man, anders als die irreführende Einbettung in den Kontext der Rechts- und Amtshilfe, hier eine Bundeskompetenz annimmt, für deren sachgerechte Wahrnehmung es dann allerdings - zumal angesichts der kaum zu leugnenden Grundrechtsrelevanz des Handelns - eines Ausführungsgesetzes bedürfte, und das zudem auf eine Kooperation mit den beteiligten Ländern eingestellt werden müsste.

$55 \mathrm{Zu}$ Recht krit. Lorse, Der Beitrag der Streitkräfte zur Neuausrichtung des Katastrophenschutzes, ZRP 2005, 6, 7 f.; Baldus, Streitkräfteeinsatz zur Gefahrenabwehr im Luftraum, NVwZ 2004, 1278, 1284; Sattler, Terrorabwehr durch die Streitkräfte nicht ohne Grundgesetzänderung, NVwZ 2004, 1286, 1287; enger Soria, (Fn. 21), DVBl. 2004, 597, 603. 
nis am Ende einer Kette von vorbereitenden Handlungen darstellt. Hier fällt normativ ins Gewicht, dass die Einbeziehung der Einrichtungen des Bundes weder von den Katastrophenschutzgesetzen der Länder zureichend aufgenommen, noch von dem Bund ausgeführt worden ist. Dabei geht es nicht darum, die jeweiligen Kompetenzen in dem Sinne zu verschleifen, dass eine Bund-Länder-Gemeinschaftskatastrophenverwaltung entsteht, sondern darum, durch die jeweiligen Ebenen hinreichende aufeinander bezogene Regelungen für das kooperative Zusammenwirken zu schaffen, die gleichwohl die Verantwortlichkeiten der jeweiligen Ebenen erhalten.

Das hier geschilderte Problem der Abstimmung von Bundes- und Landeskompetenzen gilt cum grano salis für andere Bereiche des Katastrophenschutzes. Die wichtige Standardisierung der Risikoanalysen und -bewertungen und daran anschließende Allokationsentscheidungen, ebenso wie die operative Abstimmung der einbezogenen Einrichtungen, erfordern mangels zentraler Kompetenz eines der Akteure die Zusammenarbeit zwischen Bund und Ländern, die freilich - solange man sich nicht zu einer Änderung der grundgesetzlichen Kompetenzverteilung entschließen möchte - unterhalb der Schwelle einer Gemeinschaftskatastrophenverwaltung bleiben muss. Ob dies freilich angesichts der durchaus nicht unerheblichen Grundrechtsrelevanz weiterhin in den Formen der Verwaltungsabstimmung geschehen kann, dürfte zweifelhaft sein.

\section{Grundrechtliche Anforderungen}

Grundrechte, so formuliert Eberhard Schmidt-Aßmann einleuchtend ${ }^{56}$ haben sich in den letzten 50 Jahren als die wichtigsten Struktur bildenden Kräfte für nahezu alle Gebiete der Rechtsordnung erwiesen. Im Katastrophenschutzrecht fristen sie allerdings ein Schattendasein, jedenfalls jenseits der klassischen abwehrrechtlichen Dimension. Gewiss werden in allen katastrophenschutzrelevanten Gesetzen die zum Teil intensiv die persönliche Freiheit einschränkenden Maßnahmen unter Beachtung grundrechtlicher Anforderungen durchgeformt. Dies gilt etwa für die Einschränkung der Gesundheit, des Lebens, des Eigentums und der persönlichen Fortbewegungsfreiheit. Hier äuBert sich gleichsam die polizeirechtliche Tradition dieses Rechtsgebiets.

\section{a) Grundrechtliche Schutzpflichten}

Allerdings ist diese Sichtweise unzureichend und dies tritt hervor, je mehr über die Bewältigung von Großschadenslagen infolge des Versagens primärer Vermeidungssysteme diskutiert und geeignete Strategien entwickelt werden. Dies beginnt mit einer an etwaige Risikoanalysen und -bewertungen anschließenden Differenzierung der Schutzniveaus zwischen Grundschutz und Ergänzungsschutz, findet seine Fortsetzung etwa in den Regeln der Sichtung oder Triage bei einem Großschadensereignis und endet bei der Priorisierung von Risikogruppen bei der Verteilung knapper lebensrettender Ressourcen, etwa von Impfstoffen oder antiviralen Medikamenten. Nicht anders als lange Zeit im Gesundheitsrecht, sind diese Verteilungsentscheidungen mehr oder

56 Schmidt-Aßmann, Grundrechtspositionen und Legitimationsfragen im öffentlichen Gesundheitswesen, 2001, S. 11. 
weniger implizit, werden entweder in dem Zirkel der Katastrophenschutzexperten expertokratisch als Sachnotwendigkeiten ausgewiesen oder im Eintrittsfall einer Katastrophe dem medizinischen Personal als professionelle Kompetenz zugewiesen, ohne dass man in den betreffenden Gesetzen hinreichende Anhaltspunkte dafür findet. Sie sind implizit, nicht explizit, sie werden bewusst oder unbewusst einer Öffentlichkeit sichernden Gesetzgebung entzogen.

Während in der Entwicklung des Risikoverwaltungsrechts den grundrechtlichen Schutzdimensionen eine erhebliche Struktur bildende Kraft zukam und noch zukommt, findet sich im Katastrophenschutzrecht nichts Vergleichbares. Soweit ersichtlich wird dies noch nicht einmal in Ansätzen diskutiert. Dabei kann nicht ernsthaft zweifelhaft sein, dass die grundrechtliche Schutzpflicht aus Art. 2 Abs. 2 GG Struktur bildende Funktion auch für das Katastrophenschutzrecht haben kann und muss. Wenn es Verpflichtung des Staates ist, sich schützend und fördernd vor Leben und körperliche Unversehrtheit zu stellen, dann auch und gerade, wenn die primären Präventionsinstrumente versagen oder gar nicht vorhanden sind. Allerdings mag hier noch eine andere Sperre die Diskussion blockieren. Wenn die Schutzpflichten auf den Schutz vor dem zurechenbaren und jedenfalls grundsätzlich steuerbaren Verhalten Dritter ausgerichtet sind ${ }^{57}$ dann liegt es auf den ersten Blick nahe, ihren Tatbestand zu begrenzen und konkreten Schutz vor gefährdendem Verhalten Dritter von der allgemeinen sozialstaatlichen Hilfe in Notlagen abzugrenzen und Naturkatastrophen aus dem Tatbestand der Schutzpflicht herauszunehmen. ${ }^{58}$ Auch wenn man dem folgen möchte, so ist dies einleuchtend ohnehin nur für einen Teilbereich des Katastrophenschutzes, also nicht für die zurechenbaren Schadensverläufe. Vieles von dem, was früher als unvermeidliches Schicksal angesichts der übermächtigen Natur angesehen werden konnte, wird heute zurechenbar. Die Klimaveränderung und die mit ihr einhergehenden Instabilitäten, aus denen Flutkatastrophen als typische Beispiele der Unvermeidbarkeit denn auch ihre Plausibilität ziehen, wird nicht ohne weiteres mehr dem Schicksalhaften zugerechnet werden können, das dann in den Bereich der allgemeinen sozialstaatlich motivierten Nothilfe verlagert werden könnte. Für die Katastrophen infolge des Versagens großtechnischer Systeme und terroristischer Anschläge gilt dies ohnehin. Aber auch prinzipiell mag man fragen, ob damit der alleinige und richtige Bezugspunkt für die Annahme einer Schutzpflicht gefunden ist. Vielmehr macht gerade die Wandlung des Katastrophenbegriffs und die Schwierigkeit der Zurechnung auf das Verhalten Dritter deutlich, wie künstlich die Ausscheidung von Naturkatastrophen aus dem Schutzpflichttatbestand ist. Es wäre daher auch kaum verständlich zu machen, insoweit gleichsam längs der Unterscheidung von Natur und Gesellschaft die Begründung eines Schutzanspruchs zu bejahen oder zu verneinen. ${ }^{59}$

57 Dazu grundlegend Hermes, Das Grundrecht auf Schutz von Leben und Gesundheit, 1987, S. $226 \mathrm{ff}$.

58 So explizit denn auch Hermes, (Fn. 57), S. 231 f.

59 Vgl. auch Sachs, in: Stern, Staatsrecht III/1, § 76 V 2 a â, S. 735 f.; Klein, DVB1. 1994, 489, 490; Schulze-Fielitz, in: Dreier (Hrsg.), GGK I Art. 2 II Rdn. 76; a. A. Hermes, (Fn. 57). 


\section{b) Teilhabe an knappen Ressourcen}

Darüber hinaus aber ist ein weiterer Aspekt instruktiv, insbesondere, wenn man das Gesundheitsrecht als Kontrastfolie heranzieht. Zwar besteht weithin Einigkeit, dass Art. 2 Abs. 2 GG nur in seltenen Ausnahmen einen originären Anspruch auf staatliche Leistungen begründen kann, begrenzt ohnehin auf die zum Leben unerlässlichen Güter. ${ }^{60}$ Freilich zeigt die Diskussion über Rationierungs- und Priorisierungsfragen im Gesundheitswesen deutlich eine Tendenz zur Erweiterung hin zu einem verfassungsrechtlichen Schutz einer wie auch immer zu bestimmenden Grundversorgung, ${ }^{61}$ jedenfalls aber einen derivaten Teilhabeanspruch auf gleichen Zugang zu staatlichen ärztlichen Leistungen. ${ }^{62}$

Man wird schwerlich deren Relevanz für das Katastrophenschutzrecht leugnen können. Denn es ist ja nicht zu übersehen, dass gerade in bestimmten Fällen von Katastrophen es zwar nicht um die Inklusion ins staatliche Gesundheitssystem geht, an deren Gewährleistung durch die Katastrophe sich nichts ändert, wohl aber um den elementaren Zugang zu medizinischen Leistungen in einer konkreten Situation. In diesen geht es dann buchstäblich um Leben oder Tod und damit um das nackte Existenzminimum. Der Ausbruch von Pandemien, ein von Experten für sehr wahrscheinlich erachteter Katastrophenverlauf, das Versagen großtechnischer Systeme und die Möglichkeit terroristischer Anschläge sind nur einige der Szenarien, in denen medizinische Grundversorgung erforderlich ist, deren Bestehen allerdings aber ab einer bestimmten Größenordnung schwierig sein kann. In dem Eintrittsfall aber geht es dann um Rationierungsund Priorisierungsentscheidungen, also um Differenzierungen des gleichen Zugangs zu vorhandenen medizinischen Ressourcen.

Insoweit wird man zwischen verschiedenen Ebenen und Bezugspunkten zu unterscheiden haben. Eher schwierig dürfte es sein, für das Gesamtsystem des Katastrophenschutzes Aussagen aus den Grundrechten, insbesondere den Schutzpflichten und der Leistungsdimension herzuleiten. Die hier vorhandenen Abstimmungsdefizite zwischen Bund und Ländern und einzelnen Teilbereichen, insbesondere der Frage einer hinreichenden Einbeziehung der für den Gesundheitsschutz zuständigen Behörden und des Katastrophenschutzes, überschreiten regelmäßig den Rahmen einer Operationalisierung grundrechtlicher Anforderungen. Anders dürfte dies mit den Fragen nach hinreichenden Ressourcen sein. Dies betrifft etwa Fragen nach einer hinreichenden Zahl

60 Dazu früh schon Seewald, Zum Verfassungsrecht auf Gesundheit, 1981, S. 72 ff.; vgl. auch Schulze-Fielitz, in: Dreier (Hrsg.), GGK I Art. 2 II Rdn. 96; Murswiek, in: Sachs (Hrsg.), GG, Art. 2 Rdn. 224; Starck, in: v. Mangoldt/Klein/Starck, GG, Art. 2 Abs. 2 Rdn. 212, Art. 1 Abs. 1 Rdn. 41.

61 Ausführlich dazu und zu den Ambivalenzen Schmidt-Aßmann, (Fn. 56), S. 23 ff. m.w.N.

62 Dazu Schulze-Fielitz, in: Dreier (Hrsg.), GGK I Art. 2 II Rdn. 96. Diese Diskussion hat ihren Hintergrund in der Ressourcenknappheit des beitragsfinanzierten staatlichen Gesundheitssystems und dient im Wesentlichen dazu, gleichsam den verfassungsrechtlich festliegenden Sockel einer Minimal- oder Grundversorgung zu bestimmen, bestimmt aber nicht über die Inklusion in das Gesundheitssystem, die wesentlich ohnehin für weite Teile der Bevölkerung besteht; dazu Schmidt-Aßmann, (Fn. 56), S. 23 f. 
von Impfstoffen und anderen Medikamenten oder Krankenhausplätzen, von denen heute absehbar sein dürfte, dass sie im Katastrophenfall - je nach Art und Ausmaß nicht ausreichend zur Verfügung stehen und innerhalb der notwendigen Zeit nur schwer verfügbar gemacht werden können. ${ }^{63}$ Diese Knappheit, die auf impliziten Entscheidungen beruht, führt - neben den im Katastrophenfall temporär aufgrund des jeweiligen Katastrophenverlaufs möglicherweise unvermeidbaren Knappheiten - zu Rationierungs- und Priorisierungsentscheidungen, die elementar das Recht Einzelner oder bestimmter Risikogruppen auf eine dem Existenzminimum entsprechende medizinische Versorgung einschränken. Nicht dass diese nicht gerechtfertigt werden könnten, wohl aber dass noch nicht einmal der Ansatz einer gesetzlichen oder administrativen Entscheidung darüber stattfindet, ist der eigentliche Kritikpunkt. ${ }^{64}$ Gleiches und verstärkt gilt für die Mikroebene der Allokation knapper Ressourcen. Auch hier gilt wiederum: Selbstverständlich kann es im Fall der eingetretenen Katastrophe notwendig sein, Priorisierungs- und Rationierungsentscheidungen zu treffen, aber die Grundlagen für diese Entscheidungen sind schwer erkennbar. Exemplarisch zeigt sich dies an den Entscheidungen der Behörden bei einer Pandemie, bei denen die vorhandenen antiviralen Medikamente und ggf. - sofern vorhanden - Impfstoffe nach bestimmten Kriterien für Risikogruppen vergeben werden müssen. Nichts anderes gilt im unmittelbaren Katastrophenfall bei einem Massenanfall von mehr oder weniger schwer Verletzten. Gewiss kann es hier relativ zu den in der gegebenen Situation vorhandenen Ressourcen notwendig sein, eine Sichtung und Triage nach Maßgabe der Behandlungschancen vorzunehmen. ${ }^{65}$ Aber es ist evident, dass damit das Recht eines jeden auf gleichen Zugang $\mathrm{zu}$ medizinischen Behandlungsmöglichkeiten eingeschränkt wird. Eine Grundlage für solch weit reichende Entscheidungen kann man wenn überhaupt - allenfalls in den Generalklauseln der Katastrophenschutzgesetze sehen. Hier scheint sich zu wiederholen, was auch im Gesundheitsrecht lange Zeit zu beobachten war, dass diese Entscheidungen letztlich der medizinischen Professionalität überlassen werden. Zweifelsohne stellen diese auch medizinisch zu verantwortende Entscheidungen dar, aber der Übergang von einer Normalmedizin, die den Anspruch auf gleichen Zugang zu den Ressourcen medizinischer Versorgung wahrt, zu einer nach Maßgabe von Ressourcen und Risikogruppen differenzierten Versorgung (oder Nichtversorgung) ist ebenso wenig eine medizinisch-professionelle Entscheidung wie diejenige über die Kriterien. Es ist aber schwerlich erkennbar, von wem diese anhand

$63 \mathrm{Zu}$ den Problemen Schmiedle/Sefrin, Limitierende Bedingungen der stationären Versorgung unter katastrophenmedizinischen Bedingungen, Der Notarzt, 2003, 220 ff.; Schauwecker/Schneppenheim/Bubser, Organisatorische Vorbereitungen im Krankenhaus für die Bewältigung eines Massenanfalls von Patienten, Notfall \& Rettungsmedizin, 2003, S. 596 ff.

64 Dazu schon die Anmerkung von Clausen, Neuzeitliche Organisationen angesichts gesellschaftlicher Gefahren, in: Jäger/Schimank (Hrsg.), Organisationsgesellschaft, 2005, S. 493, 501.

65 Dazu Bloß, Zwang zur Selektion, DÄBl 101 (2004), S. 2216 ff; Sefrin, Sichtung als ärztliche Aufgabe, DÄBl 102 (2005), S. 1424 ff. 
welcher Kriterien und innerhalb welcher Verantwortungsstruktur gefällt wird. ${ }^{66}$ So zeigt sich in der nationalen Pandemieplanung sehr deutlich, dass unterschiedliche Ansatzpunkte für die Bildung von Risikogruppen bestehen, je nachdem, ob politisch-soziale Aspekte, epidemiologisch-dynamische Aspekte oder die maximale Reduktion der Krankheitslast in den Vordergrund gestellt werden. Innerhalb der zweiten Gruppe kann wiederum unterschieden werden nach Risiko des tödlichen Ausgangs innerhalb bestimmter Altersgruppen, Verhinderung einer möglichst großen Anzahl von Toten oder maximalem wirtschaftlichen Nutzen. Je nach gewähltem Kriterium unterscheiden sich die prioritären Risikogruppen erheblich. ${ }^{67} \mathrm{Zu}$ Recht betont der Influenza-Pandemieplan, dass die Kriterien transparent und begründet sein müssen und die Entscheidungen bundeseinheitlich und im gesellschaftlichen Konsens getroffen werden müssen. ${ }^{68}$ Ebenso deutlich ist, dass dies keine medizinisch-wissenschaftliche Entscheidung ist, sondern eine evident politische und evident grundrechtsrelevante. Hinzu kommt, dass die Prioritätenliste von den vorhandenen Ressourcen und deren Verteilung abhängig ist. Dies alles erfordert eine gesetzliche Grundlage, die derzeit nicht erkennbar ist. Der nahe liegende Einwand, diese könne aufgrund der Ungewissheit über den konkreten Verlauf nicht mehr als eine Generalklausel sein, die wenig an Entscheidungssicherheit vermittelt, verschlägt nicht. Dem lässt sich durch eine hinreichend flexible Gestaltung Rechnung tragen. Gleiches gilt dann für die Einbeziehung der »Nationalen Pandemiekommission«, die im Ernstfall nach der Planung Präzisierungen der Prioritätenliste aufgrund von epidemiologischen Gesichtspunkten vornehmen soll. ${ }^{69}$ Organisation, Verfahren und Kriterien einer solchen Priorisierungsstrategie wären allererst gesetzlich zu regeln. ${ }^{70}$ Diese nur pars pro toto vorgestellten Beispiele zeigen, dass zwar Anstrengungen unternommen werden, die nötigen Ressourcen zur Verfügung zu stellen, aber dass ein evidentes Problem die fehlenden rechtlichen Strukturen sind, die diese Entscheidungen stützen und verantwortbar machen.

\section{Die Einbeziehung der Öffentlichkeit}

Nichts anderes gilt für die Einbeziehung der Öffentlichkeit. Wenn - das Risikoverwaltungsrecht zeigt es - das Grundrecht auf Schutz des Lebens und der körperlichen Unversehrtheit sich auch und vor allem in Organisation und Verfahren äußert, dann fällt

66 Die zunächst nahe liegende Möglichkeit, diese Entscheidung selbst schon in der Feststellung des Katastrophenfalles durch die zuständige Katastrophenschutzbehörde sehen zu wollen, scheidet schon deshalb aus, weil sie nichts darüber aussagt, ob insoweit hinreichende Ressourcen vorhanden und wie sie einzusetzen sind. In der Sache wird kaum etwas anderes übrig bleiben, als sie dem Leitenden Rettungsarzt zuzuweisen, aber dafür bedürfte es einer Grundlage und der Angabe von Kriterien; derzeit ist diese Grundlage nicht erkennbar.

67 Nationaler Influenzapandemieplan Teil II. Analysen und Konzepte. Bericht der Expertengruppe »Influenza-Pandemieplanung « am Robert Koch-Institut, 2005, S. 35 ff.

68 Nationaler Pandemieplan, (Fn. 67), S. 35.

69 Nationaler Influenza-Pandemieplan, (Fn. 67), S. 37.

70 Geschehen ist dies für die Ständige Impfkommission beim Robert Koch-Institut (vgl. § 20 Abs. 2 IfSG) wegen der weitreichenden Wirkung ihrer Empfehlungen. 
die Arkanpraxis des Katastrophenschutzes besonders ins Auge. So sehr immer wieder die Notwendigkeit der Einbeziehung der Bevölkerung in den Katastrophenschutz unter dem Gesichtspunkt der Aufklärung über Risiken und Selbstschutzmöglichkeiten gefordert ist, so wenig sind bisher geeignete Formen der Einbeziehung derer, die allererst spätere Opfer sein können, vorgesehen. ${ }^{71}$ Erst in vorsichtigen Ansätzen zeigen sich hier Veränderungen, die ihre Impulse neben den aus dem europäischen Recht folgenden Vorgaben vor allem aus Querschnittsbereichen beziehen, in denen die Öffentlichkeit schon seit langem einen anderen Status hat. Exemplarisch ist dafür etwa die Neuregelung über die Festlegung von Überschwemmungsgebieten (Art. 31 b Abs. 2 WHG) ${ }^{72}$, für die nicht nur behördliche Informationspflichten festgelegt sind, sondern auch ein Verfahren der Öffentlichkeitsbeteiligung vorgesehen ist. Zu Recht wird insoweit der Zusammenhang zwischen Öffentlichkeitsinformation und -beteiligung und der Sensibilisierung der Bevölkerung für die Belange des vorbeugenden Katastrophenschutzes hervorgehoben. ${ }^{73}$ Dem entspricht es, wenn korrespondierend zu Verpflichtungen der Eigenvorsorge und Schadensminimierung, wie sie etwa neuerdings in $\S 31$ a Abs. 2 WHG vorgesehen sind, Informationspflichten der Behörden über Gefahren und geeignete Vorsorgemaßnahmen festgelegt werden ( $\$ 31$ a Abs. 3 WHG). ${ }^{74}$ Risikoanalysen und -bewertungen, sowie daran anschließende Ressourcenplanungen werden jedenfalls dann, wenn sie grundrechtlich sensible Rechtspositionen ausgestalten, kaum ohne eine Beteiligung der Betroffenen oder Öffentlichkeit auskommen können.

\section{Ausblick}

Damit sind nur einige der möglichen verfassungsrechtlichen Fragen angesprochen, andere bedürften der Untersuchung. Die Einbindung in die europäische Dimension wäre eine weitere, die von manchen der Akteure schon als Option einer von den bundesstaatlichen Abstimmungsproblemen befreienden Modernisierung angesehen wird. Auf Dauer gesehen wird man schwerlich einer rechtsstaatlichen Durchformung des Katastrophenschutzrechts ausweichen können, wenn der Befund einer stärkeren Verbindung von Prävention und Katastrophenschutz und die Tendenzen zu einem risikodiffe-

71 Weniges zeigt die Tradition so deutlich, wie die verbreiteten Vorbehalte, Gefahrenhinweiskarten überschwemmungsgefährdeter Gebiete zu veröffentlichen; vgl. dazu Petrow/Thieken/Kreibich/Merz, Vorsorgende Maßnahmen zur Schadensminderung, in: Deutsches Komitee für Katastrophenvorsorge e.V. (DKKV), Hochwasservorsorge in Deutschland. Lernen aus der Katastrophe 2002 im Elbegebiet, 2003, S. 40; anders jetzt aber etwa $\S 31$ c, d WHG in der Fassung des Art. 1 Nr. 4 des Gesetzes zur Verbesserung des vorbeugenden Hochwasserschutzes v. 31. Mai 2005 (BGBl. I S. 1224).

72 Fassung des Art. 1 Nr. 4 des Gesetzes zur Verbesserung des vorbeugenden Hochwasserschutzes v. 31. Mai 2005 (BGBl. I S. 1224).

73 Rechenberg, Vom Fünf-Punkte-Programm zum Hochwasserschutz-Artikelgesetz - Hochwasserschutz aus rechtlicher Sicht, S. 5 (Vortragsmanuskript).

$74 \mathrm{Vgl}$. dazu Art. 1 Nr. $4 \S 31$ a Abs. 3 des Gesetzes zur Verbesserung des vorbeugenden Hochwasserschutzes v. 31. Mai 2005 (BGBl. I S. 1224); dazu Rechenberg, Vom FünfPunkte-Programm zum Hochwasserschutz-Artikelgesetz - Hochwasserschutz aus rechtlicher Sicht, S. 4 (Vortragsmanuskript). 
renzierten Katastrophenschutz richtig beobachtet sind. Die darin eingeschlossenen Priorisierungs- und Verteilungsentscheidungen, aber auch die Grundfrage, welche Ressourcen für welches Schutzziel aufgewendet werden sollen, sind solche, die nicht nur eine öffentliche Debatte, sondern auch demokratische Entscheidungen erfordern. Wie in anderen Bereichen auch mag der nahe liegende Einwand lauten, dass explizite Entscheidungen zu gesellschaftlichen Konflikten und einer überflüssigen oder gar schädlichen Verrechtlichung führen. ${ }^{75}$ Wie immer man sich dazu stellen mag, sub specie der Wesentlichkeitslehre lässt sich schwerlich bezweifeln, dass gerade im vorbeugenden Katastrophenschutz parlamentarische Entscheidungen unausweichlich sein dürften. Das für gewöhnlich zu findende Argument der Nichtregelbarkeit der Katastrophe verfehlt die Sache, denn darum geht es nicht. Es geht darum, eine für den Katastrophenfall angemessene Unterstützungs- und Hilfsstruktur zu entwickeln. Deren Bedeutung für die Rechtsgüter des Einzelnen dürfte nicht ernsthaft im Streit stehen. Aber auch für das politische System birgt die Latenz, in der der Katastrophenschutz gehalten wird, nicht unerhebliche Risiken der Delegitimation, wie die derzeitige Diskussion in den USA in Folge des Hurrikans Katrina zeigt. Dabei geht es weniger um die Zurechnung auf Klimawandel oder nicht, es geht vor allem um den Zustand des Katastrophenschutzes und die darin eingeschriebenen Entscheidungen und Leitbilder. ${ }^{76}$ Katastrophen fegen in großer Geschwindigkeit die gesellschaftlichen Routinen und Erwartungen beiseite und eröffnen einen Blick auf gesellschaftliche Grundentscheidungen, die vorher latent gehalten worden sind. Von daher spricht auch aus der Sicht des politischen Systems viel dafür, einer offenen Diskussion um die damit aufgeworfenen Entscheidungen nicht auszuweichen. Die geeigneten (rechtlichen) Formen zu finden, bleibt eine zu lösende Aufgabe, die freilich eine detailliertere Bestandsaufnahme der formellen und informellen Strukturen voraussetzt, als sie hier möglich war. Katastrophenschutz ist - wenn nicht alles täuscht - eine zunehmend wichtigere und voraussetzungsvollere Aufgabe, deren Leitbild nicht die paternalistische Daseinsvorsorge durch Staat und Experten sein kann, sondern die als civil protection verstanden, auch eine gesellschaftliche Aufgabe ist. Laien sind die Bürger immer nur, solange die Katastrophe nicht eingetreten ist. Danach werden sie ohnehin zu Experten - der Sicherung ihrer eigenen Existenz. Sie dazu in die Lage zu versetzen und sie darin zu unterstützen, wäre vielleicht ein angemessenes Leitbild.

$75 \mathrm{Zu}$ vergleichbaren Argumenten im Gesundheitsrecht Schmidt-Aßmann, (Fn. 56), S. 59 ff.

76 Nochmals am Beispiel von Katrina: Ein Blick in den Superdome von New Orleans legt die enormen Stratifizierungen der amerikanischen Gesellschaft offen, so wie die Bilder von Opfern, die von bewaffneten Einsatzkräften am Verlassen des Orts der Katastrophe gehindert werden, Leitbilder des Katastrophenschutzes offenbaren, bei denen die Opfer als Bedrohung einer Ordnung wahrgenommen werden, die ihnen elementaren Schutz nicht gewähren kann oder will. 